\title{
NICHE PARTITIONING ALONG AN ENVIRONMENTAL GRADIENT*
}

\author{
ISAAC KLAPPER ${ }^{\dagger}$, JACK DOCKERY ${ }^{\ddagger}$, AND HAL SMITH ${ }^{\S}$
}

\begin{abstract}
Biological systematics studies suggest that species are discretized in niche space. That is, rather than seeing a continuum of organism types with respect to continuous environmental variations, observers instead find discrete species or clumps of species, with one clump separated from another in niche space by a gap. Here, using a simple one dimensional model with a smoothly varying environmental condition, we investigate conditions for a discrete niche partitioning instability of a continuously varying species structure in the context of asexually reproducing microbes. We find that significant perturbation of translational invariance is required for instability, but that conditions for such perturbations might reasonably occur, for example, through influence of boundary conditions.
\end{abstract}

Key words. niche partitioning, microbial ecology

AMS subject classifications. 92D40, 34E10, 34C60

DOI. $10.1137 / 14095786 \mathrm{X}$

\section{Introduction.}

1.1. Background. Characterization of plant and animal ecological structures via species classification has a long and distinguished history. More recently, cataloging microbial species, partly as a consequence of rapidly improving access to molecular sequencing technology, has become increasingly popular as well. Despite the ubiquity and importance of microbes, however, theory for ecological characterization of prokaryotic microbial communities is still relatively underdeveloped and, as a consequence of asexual reproduction among other things, may be rather different than theory developed for plants and animals [35]. It is generally suspected that, via their rapid reproduction rate combined with a variety of genetic manipulation capabilities (using enhanced mutation, horizontal gene transfer, etc.), inhabitants of microbial ecosystems are able to adapt comparatively quickly to their environment in comparison to multicellular organisms. Understanding of the consequences for the resulting ecological structures, though, is limited.

We might suppose at least in some instances that microbial communities can adapt to their environments on shorter times than the time scale of secular variation of their environments. Then community structure could be determined in large part by relatively straightforward competitive processes. (External stresses like predation might also be important but here we suppose such influences to affect all species equally. It should be noted though that when predation is present, Turing-like instabilities may also be possible [27].) In well-mixed systems like chemostats [46], where environmental conditions are effectively spatially uniform, there are questions as to

* Received by the editors February 20, 2014; accepted for publication (in revised form) June 23, 2014; published electronically September 25, 2014. This work was supported by NSF awards DMS1022836, DMS-091844, and MCB-1158531.

http://www.siam.org/journals/siap/74-5/95786.html

$\dagger$ Department of Mathematics, Temple University, Philadelphia, PA 19122 (klapper@temple.edu).

${ }^{\ddagger}$ Department of Mathematical Sciences, Montana State University, Bozeman, MT 59717 (dockery@math.montana.edu).

${ }^{\S}$ School of Mathematical \& Statistical Sciences, Arizona State University, Tempe, AZ 85287 (halsmith@asu.edu). 
why there should be significant species variation at all, the so-called paradox of the plankton [23]. Here well-mixed means that the mixing time is small in comparison to other time scales, especially the growth time. The case that the environment isn't homogenized but the microorganisms are highly motile (so as to be able to sample across the environment on a relatively short time scale) is likely similar. However in many instances, microbial ecosystems are not well-mixed in either of these senses. As a consequence, different organisms may be effectively exposed to different environmental histories depending on their location within the community. Thus in contrast to the well-mixed case, in a stable, continuously (in space) varying environment, one might expect rapid (in space) variation in species, each adapted to its local conditions, even if the environment varies relatively slowly (in space). In the extreme instance of no microbial mobility at all, spatial location becomes a parameter and species structure might in theory vary continuously as adaptation to the local microenvironment occurs pointwise. If there is a paradox here, it is instead a question of why there are not more species rather than less.

A related problem, called the "paradox of the clumps" [38], refers to field observations of niche separation in a well-mixed domain for which data are catalogued by a niche variable, for example, organismal body size, resulting in apparently nonhomogeneous species distribution in the relevant niche characteristic. Studies of this phenomenon may allow species to interact with nearby (in niche coordinates) ones through a Lotka-Volterra competition model. Typically, competitive mechanisms of all sorts are combined under the umbrella of a single, phenomenologically defined competition kernel, often of Gaussian or similar form. Depending on choice of competition kernel, such models predict instability (or not) of homogeneous niche space species structure leading to clumping in niche space (or not). Which occurs is determined by choice of the competition kernel, with fairly sensitive dependence on such details as tail decay rates $[19,34,39]$. Related models have included environmental changes in space in the form of spatially dependent Lotka-Volterra carrying capacities [26, 36]. Again, however, results depend sensitively on kernel details. As a consequence, we opt to move away from the Lotka-Volterra dynamics and, rather, choose a particular competition mechanism, namely, competition for space. Space is a key resource in many communities, particularly microbial biofilms where suitable habitats can be strictly confined by steep environmental gradients arising most typically as a consequence of reaction-diffusion effects. As will be seen, we find in this case that results do not appear to be sensitive to small parametrization details (such as tail decay rates). A second central component of the model presented here is spatial transport in the form of diffusive drift. Often, niche space based models also include diffusive drift in niche space itself as a way to include evolutionary effects. Here, however, diffusion acts in real space only.

Whether it is advantageous for an organism to be mobile in a spatially heterogeneous but temporally homogeneous environment has been the subject of a considerable body of research. While this question is not central to our focus here, we note that Hastings [20] was among the first to seek answers through mathematical models. He showed that so-called unconditional mobility, random mobility independent of environmental conditions, is disfavored. Later work of Dockery et al. [10] corroborated and extended this conclusion. See the review chapter by Cantrell, Cosner, and Lou [5] for more recent work. Though we allow random mobility here, relative fitness with respect to mobility amplitude is not considered.

Despite the microscopic dimensions of each individual inhabitant, microbial communities can extend over noticeable distances, e.g. lake and stream beds, length scales 


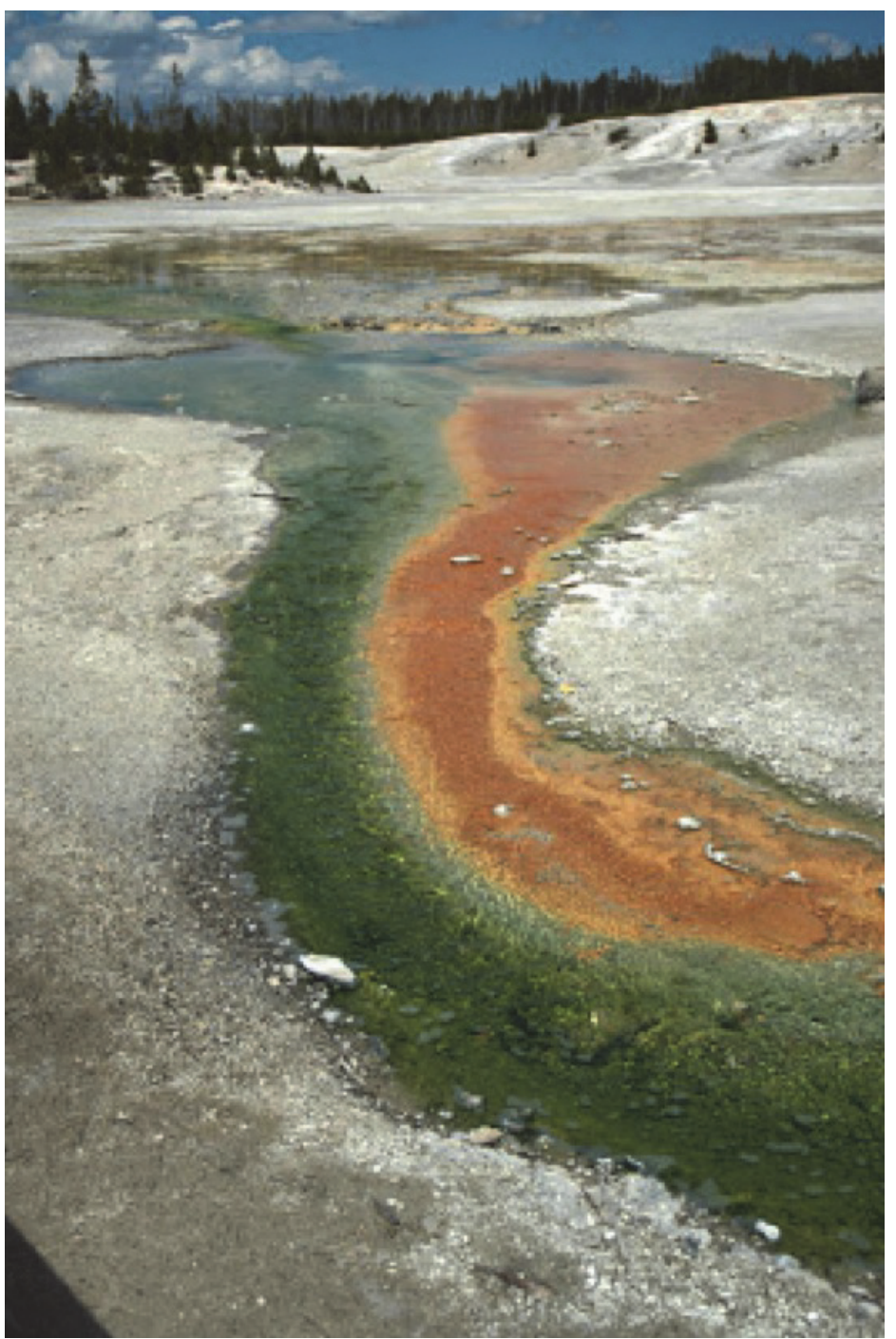

Fig. 1. Microbial mat in an effluent channel, Pinwheel Spring in Norris Basin, Yellowstone National Park. Water from two different sources, each at its own temperature and with its own chemistry, runs together into the same effluent channel. Species makeup changes rapidly (green indicates presence of photoautotrophs, red indicates presence of iron oxidizing chemotrophs) at the interface between the two runoffs, as suggested by color change in the cross channel direction. Photo credit: Eileen Nauman.

large enough so that environmental conditions can change significantly. Further, as mentioned, as a consequence of the fact that for sessile communities much and sometimes all of the environment is not well-mixed, it is typical that community related diffusion-reaction balances induce spatial variation by themselves, even over distances of the order of microns through reaction-diffusion related formation of stratified microenvironments $[6,25]$. This self-induced source of heterogeneity tends to lead to sharp environmental variation and, consequently, niche boundaries [47]. Related effects, though not necessarily self-induced, can be seen in microbiological communities, e.g., [2] and Figure 1. 
However, niche partitioning is observed even in slowly varying (in space) environments where appeal cannot be made to sharp transitions. By slowly varying, we mean that organism displacements of the sort resulting from mobility do not result in exposure to sharply variant environmental conditions on growth related time scales or faster. Referring to Figure 1, for example, this sort of species variation would be observed in the down-channel direction, not (necessarily) the cross-channel one. Here, then, we consider the possibility of a spatially varying environment with some organismal mobility and, in particular, we examine the tendency for niche separation in a community of mobile microbial species in a slowly varying (in space) environment. Using a one dimensional model environment with a slowly varying environmental condition (e.g., temperature or light) and a simple model microbial community system, we will work to obtain insight into issues of continuous versus discrete species structures. Are there mechanisms by which discrete species structure spontaneously develops in slowly varying environments? To rule out effects of inherently favorable local environmental conditions, we at first address this question using a linearly varying environment together with a biological response which is translation invariant in the sense that no particular environmental condition is inherently favorable to others. This is not necessarily a representative assumption - for example, in reality, obviously, temperatures that are too cold or too hot cannot support life at all. However its use allows study of the problem in a more "pure" way. Subsequently, though, we allow favorable local environments in several different ways and study the resulting species structure when some of the realities of actual environments are present.

2. Two species. We first examine the interaction of two species with a view towards understanding when they can and cannot coexist. The aim is to inform results for a continuously varying species ecology model that will follow. In particular, we ask how do very similar species compete? With that motivation, consider, then, two species, with volume fractions $X_{1}\left(x, t ; \tau_{1}\right)$ and $X_{2}\left(x, t ; \tau_{2}\right)$, on domains $x \in[a, b]$ or $x \in$ $(-\infty, \infty)$. Here volume fraction can be interpreted to mean the occupied percentage of an infinitesimal one dimensional volume $d x$. We assume that $X_{1}+X_{2}=1$ for all $x$ with the implication that the two species compete for space. Note also that only solutions with $0 \leq X_{1}, X_{2} \leq 1$ are of interest. On the finite domain, application of noflux boundary conditions is natural. We introduce a spatially varying environmental condition $T=T(x)$, e.g., temperature. Parameters $\tau_{1}$ and $\tau_{2}$ index the response to this environmental condition as explained below.

Populations of species 1 and 2 change in time according to

$$
\begin{aligned}
& X_{1, t}=r\left(f\left(T ; \tau_{1}\right)-c(x, t)\right) X_{1}+D X_{1, x x}, \\
& X_{2, t}=r\left(f\left(T ; \tau_{2}\right)-c(x, t)\right) X_{2}+D X_{2, x x},
\end{aligned}
$$

where $\mathrm{r}$ is a growth rate coefficient and $f(T ; \tau)$ is the dimensionless environmental response of species $\tau$ to condition $T$ ( $T$ and $\tau$ have the same units). The function $c(x, t)$ is a loss rate determined so as to enforce the constraint $X_{1}+X_{2}=1$ and can be computed by adding these equations to obtain

$$
c(x, t)=f\left(T ; \tau_{1}\right) X_{1}+f\left(T ; \tau_{2}\right) X_{2} .
$$

The supplied function $f(T ; \tau)$ distinguishes one species from another through its $\tau$ dependence; $\tau$ can, for example, indicate the optimum environmental condition for growth, though not necessarily. We will assume that $f$ is integrable with $f>0$ and as smooth as necessary. As an example, $f$ could be chosen to be of Gaussian form 
$f(T ; \tau)=C \exp \left(-(\tau-T)^{2} / \gamma\right)$, indicating that a particular species with designation $\tau$ grows fastest at environmental condition $T=\tau$. More generally, a form $f=$ $f(T-\tau)$ indicates that species response translates with $T-\tau$ though not requiring that response be symmetric in the sign of $T-\tau$. We will suppose that $T(x)=\alpha x$ so that no one local environmental profile is distinguishable from another in the sense that environment changes uniformly with translation, i.e., $T^{\prime}(x)$ is constant. The units for the environmental condition are arbitrary, so we choose them in such a way that $\alpha=1$. We can go ahead, in fact, and conflate units of the environmental condition with length units. For example, in the case where the environmental condition is temperature, then position and temperature are effectively interchangeable.

Using $X_{2}=1-X_{1}$ in (2.1) and renaming $X_{1}$ by $X$, then

$$
X_{t}=\beta(x) r X(1-X)+D X_{x x}
$$

where $\beta(x)=f\left(x ; \tau_{1}\right)-f\left(x ; \tau_{2}\right)$. We are particularly interested in characterizing equilibrium $\left(X_{t}=0\right)$ solutions of (2.2). Note the extinction solutions $X=1$ (i.e., $\left.X_{1}=1, X_{2}=0\right)$ and $X=0$ (i.e., $X_{1}=0, X_{2}=1$ ). Defining an energy functional

$$
E[X](t)=\int\left[\beta(x) r\left(\frac{X^{3}}{3}-\frac{X^{2}}{2}\right)+\frac{1}{2} D X_{x}^{2}\right] d x,
$$

a short computation shows that, for $X$ a solution of (2.2),

$$
\frac{d}{d t} E=-\int X_{t}^{2} d x \leq 0 .
$$

For the solutions $X=0$ and $X=1$, we have $E[0]=0$ and $E[1]=-(1 / 6) \int \beta(x) r d x$. Thus $X=0$ is more energetically favorable when $\int \beta(x) d x<0$ and $X=1$ is more energetically favorable when $\int \beta(x) d x>0$. Given a solution $X(x, t ; \tau)$ of $(2.2)$, we consider an approximate perturbation $X+\hat{X}$, where $\hat{X}$ satisfies

$$
\hat{X}_{t}=\beta(x) r(1-2 X) \hat{X}+D \hat{X}_{x x},
$$

the linearization of (2.2). Note for the solution $X=0$ we see that $\hat{X}_{t}=\beta r \hat{X}+D \hat{X}_{x x}$ so that, if $\int \beta(x) d x>0$ then $X=0$, is unstable to, for example, any constant positive perturbation. Likewise for the solution $X=1$ we see that $\hat{X}_{t}=-\beta r \hat{X}+D \hat{X}_{x x}$ so that, if $\int \beta(x) d x<0$, then $X=1$ is unstable to, for example, any constant negative perturbation. Stability of the solution $X=0$ when $\int \beta(x) d x<0$ and of the solution $X=1$ when $\int \beta(x) d x>0$ is more subtle and depends on the relative importance of growth and diffusivity. Note for example that, for $D=0$ and $\beta(x)$ not single signed, neither $X=0$ nor $X=1$ is linearly stable. Interestingly, when $\int \beta(x) d x=0$ and $D \neq 0$, neither solution $X=0$ nor $X=1$ is stable.

To be precise, consider (2.1) for $x \in[-L, L]$ with Neumann boundary conditions, where $f\left(x ; \tau_{i}\right) \geq 0, i=1,2$, are such that no dominance relation holds on $[-L, L]$, i.e., $\beta(x)$ takes both positive and negative values on $[-L, L]$. Without loss of generality we may assume that, on average, species two is not inferior in fitness to species one:

$$
\int_{-L}^{L} f\left(x ; \tau_{1}\right) d x \leq \int_{-L}^{L} f\left(x ; \tau_{2}\right) d x
$$

i.e., $\int_{-L}^{L} \beta(x) d x \leq 0$. If strict inequality holds in (2.4), define

$$
\lambda(\beta)=\inf _{\psi \in S} \frac{\int_{-L}^{L}\left(\psi^{\prime}(x)\right)^{2} d x}{\int_{-L}^{L} \beta(x) \psi^{2}(x) d x},
$$


where

$$
S=\left\{\psi \in W^{1,2}(-L, L): \int_{-L}^{L} \beta(x) \psi^{2}(x) d x>0\right\} .
$$

Note that $S$ is not empty since $\beta$ attains positive values and that constant functions do not belong to $S$ due to our assumption that strict inequality holds in (2.4). Thus, the numerator of (2.5) cannot vanish. According to [30], $\lambda(\beta)>0$ and the infimum is attained. Recall that equations (2.1) have trivial solutions $X_{1} \equiv 1, X_{2} \equiv 0$ and $X_{1} \equiv 0, X_{2} \equiv 1$. We say that $\left(X_{1}, X_{2}\right)$ is a nontrivial equilibrium if $X_{i}(x) \geq 0$ but $X_{i}$ is not identically zero, and $X_{1}(x)+X_{2}(x)=1$ holds for all $x$. Note that, in fact, by maximum principle arguments, $X_{i}(x)>0$ holds for any nontrivial equilibrium.

Theorem 2.1 (Thm. 3.2, Remark 3.3 in [30]; Thms. 3.1 and 5.1 in [14]). The following hold.

(a) If strict inequality holds in (2.4), then

(i) if $r / D \leq \lambda(\beta)$, then the trivial equilibrium $X_{1} \equiv 0, X_{2} \equiv 1$ is globally asymptotically stable;

(ii) if $r / D>\lambda(\beta)$, then there is a unique nontrivial equilibrium which attracts all nontrivial initial data.

(b) If equality holds in (2.4), then there exists a unique nontrivial equilibrium which attracts all nontrivial initial data.

If species two is, on average, superior to species one, then it excludes species one at all locations provided that $D$ is not too small $(r / D \leq \lambda(\beta))$; otherwise $(r / D>\lambda(\beta))$, the two species coexist. In the case that the two species have, on average, identical fitness, then they coexist. An interesting point is that even if species two has, on average, superior growth fitness to species one, it is still obliged to share habitat with species one if $\sqrt{D / r}$ is sufficiently small. Note that the length scale $\sqrt{D / r}$ measures, roughly, the distance a disturbance is spread via diffusion over the growth time scale. One can then interpret the coexistence case (a)(ii) of Theorem 2.1 in two ways: (1) if diffusive transport is small enough, then microbes are better able to remain in the most favorable locations where they have a growth advantage, allowing persistence of both species even if one is on spatial average better adapted to the environment than the other. On the other hand, if diffusive transport is sufficiently large, then microbes are forced to sample disadvantageous regions and then the species with greater average fitness can exclude the lesser. (2) An alternative view is that if the length scale $\sqrt{D / r}$ is small relative to the distance between optimal regions of species one and two, then diffusive transport is relatively ineffective as a competitive tool and both species can cooccur. On the other hand, if that length scale is relatively large, then the species with the larger average can use diffusive transport to invade the lesser species' favored regions faster than growth can counterbalance.

2.1. Asymptotics. While Theorem 2.1 describes conditions for coexistence, it doesn't say much about the nature of coexisting solutions. In particular, in preparation for studying coexistence of a continuum (in $\tau$ ) of species, we would like to know more about the form of solutions when species are asymptotically close together (in $\tau)$. Let, then, $\tau_{0}=\left(\tau_{1}+\tau_{2}\right) / 2, \epsilon=\tau_{0}-\tau_{2}$ (so $\left.\tau_{1}=\tau_{0}+\epsilon, \tau_{2}=\tau_{0}-\epsilon\right)$. In anticipation of the continuous species problem, let $\epsilon \rightarrow 0$, with the motivation of studying whether two very "nearby" species can coexist stably or not and, if so, how. We expand as

$$
\begin{aligned}
X & =X^{(0)}+\epsilon X^{(1)}+\epsilon^{2} X^{(2)}+O\left(\epsilon^{3}\right), \\
\beta & =f\left(x ; \tau_{0}+\epsilon\right)-f\left(x ; \tau_{o}-\epsilon\right)=2 f_{\tau}\left(x ; \tau_{0}\right) \epsilon+O\left(\epsilon^{3}\right),
\end{aligned}
$$

Copyright $@$ by SIAM. Unauthorized reproduction of this article is prohibited. 
and assume $f_{\tau}\left(x ; \tau_{0}\right) \neq 0$ identically, i.e., that the two species are asymptotically distinguishable to first order. We are interested in the long time behavior of $X$, so we set initial conditions $X^{(0)}(x, 0)=X(x, 0)$ and $X^{(1)}(x, 0)=X^{(2)}(x, 0)=0$ and consider large $t$ behavior.

2.1.1. Zeroth order. To zeroth order,

$$
X_{t}^{(0)}=D X_{x x}^{(0)} .
$$

On a finite spatial interval, $X^{(0)}$ will approach a constant $C=\left\langle X^{(0)}(x, 0)\right\rangle$ on a diffusion time scale, where $\langle\cdot\rangle$ denotes averaging with respect to $x$. We will see below that only certain values of $C$ are consistent with the proposed asymptotic expansion for long times, with the implication over long times that the growth term $\beta r X(1-X)$ will asymptotically drive $\langle X\rangle$ to one of those distinguished values.

2.1.2. First order. The first order problem is

$$
X_{t}^{(1)}=2 r f_{\tau}\left(x ; \tau_{0}\right) X^{(0)}\left(1-X^{(0)}\right)+D X_{x x}^{(1)}
$$

which will tend towards an equilibrium (if stable) determined by

$$
0=2 r f_{\tau}\left(x ; \tau_{0}\right) C(1-C)+D X_{x x}^{(1)} .
$$

In the case of no-flux boundary conditions, or with $X_{x} \rightarrow 0$ as $|x| \rightarrow \infty$ for an unbounded domain, we can average (2.8) to obtain the Fredholm condition

$$
0=C(1-C)\left\langle f_{\tau}\left(x ; \tau_{0}\right)\right\rangle .
$$

Note thus that if $\left\langle f_{\tau}\left(x ; \tau_{0}\right)\right\rangle \neq 0$, indicating that one species has an asymptotic overall advantage over the other, then $C=0$ or $C=1$. Averaging (2.7) to obtain

$$
\left\langle X^{(1)}\right\rangle_{t}=2 r\left\langle f_{\tau}\left(x ; \tau_{0}\right)\right\rangle C(1-C)
$$

it becomes evident that, for $C \neq 0$ or $1,\left\langle X^{(1)}\right\rangle$ will increase secularly if $\left\langle f_{\tau}\left(x ; \tau_{0}\right)\right\rangle>0$ and decrease secularly if $\left\langle f_{\tau}\left(x ; \tau_{0}\right)\right\rangle<0$. In either case the asymptotic expansion breaks down, but nevertheless we infer that if $\left\langle f_{\tau}\left(x ; \tau_{0}\right)\right\rangle>0$ (overall advantage to species 1) then the solution $X=0$ is unstable and $X$ will tend to 1 generally, and if $\left\langle f_{\tau}\left(x ; \tau_{0}\right)\right\rangle<0$ (overall advantage to species 2 ) then the solution $X=1$ is unstable and $X$ will tend to 0 . This observation follows from directly averaging (2.2) as well.

If $\left\langle f_{\tau}\left(x ; \tau_{0}\right)\right\rangle=0$ (no overall advantage for either species) and $C \neq 0$ or 1 , we need to proceed to second order in $\epsilon$ to obtain a value for $C$. Note that if $f=f(x-\tau)$, then $f_{\tau}=-f_{x}$ so that $\left\langle f_{\tau}\left(x ; \tau_{0}\right)\right\rangle=\left(f\left(a-\tau_{0}\right)-f\left(b-\tau_{0}\right)\right) /(b-a)$. On $(-\infty, \infty)$ this quantity will be zero under the condition $f \rightarrow 0$ as $|x| \rightarrow \infty$, but on a finite interval it generally will not be zero, with sign determined by the relative advantage of conditions at the interval endpoints. That is, as a consequence of boundary effects, for most choices of $\tau_{0},\left\langle f_{\tau}\left(x ; \tau_{0}\right)\right\rangle \neq 0$ so that $C=0$ or 1 necessarily so that the two species will not coexist.

2.1.3. Second order. In the case $\left\langle f_{\tau}\left(x ; \tau_{0}\right)\right\rangle=0$ and $C \neq 0$ or 1 , we consider the second order problem

$$
X_{t}^{(2)}=2 r f_{\tau} X^{(1)}\left(1-2 X^{(0)}\right)+D X_{x x}^{(2)}
$$

Copyright $@$ by SIAM. Unauthorized reproduction of this article is prohibited. 
which will tend to an equilibrium (if stable)

$$
0=2 r f_{\tau} X^{(1)}(1-2 C)+D X_{x x}^{(2)} .
$$

Averaging, we obtain the Fredholm condition

$$
0=\left\langle f_{\tau} X^{(1)}\right\rangle(1-2 C) .
$$

Note that at steady state, from (2.8) upon multiplying by $X^{(1)}$ and averaging,

$$
r\left\langle f_{\tau} X^{(1)}\right\rangle C(1-C)=D\left\langle\left(X_{x}^{(1)}\right)^{2}\right\rangle .
$$

Since $C \neq 0$ or 1 , if $\left\langle f_{\tau} X^{(1)}\right\rangle=0$ at steady state then $X^{(1)}$ will be a constant (in $x$ ) at steady state. From (2.7), however, this requires $X^{(0)} \rightarrow 0$ or 1 or $f_{\tau}\left(x ; \tau_{0}\right)=0$, contrary to assumptions. Hence we can conclude that, at steady state, if $C \neq 0$ or 1 then $\left\langle f_{\tau} X^{(1)}\right\rangle \neq 0$ and thus, from (2.11), that $C=1 / 2$. That is, species 1 and 2 coexist equally to zeroth order. Recall also that $C=\left\langle X^{(0)}(x, 0)\right\rangle$. If $\left\langle X^{(0)}(x, 0)\right\rangle \neq 1 / 2$ then $X^{(2)}$ will increase or decrease secularly.

In summary, asymptotics for the two species model suggest that the continuous species model will not indicate species clumping unless the uniform favorability of environmental response is somehow broken. Rather, a translationally invariant solution should be expected; when two asymptotically similar species are able to coexist, they spread equally through the domain to zeroth order as opposed to creating a sharp boundary between domains where one or the other is dominant.

2.2. Numerics: Two and three species. Note that the assumption that $\beta r=$ $2 r f_{\tau}\left(x ; \tau_{o}\right) \epsilon+O\left(\epsilon^{2}\right)$ is small breaks down at fixed $\epsilon$ for large growth rate $r$. To investigate, we compute solutions at fixed $\epsilon$ of the two species model

$$
\begin{aligned}
& X_{1, t}=r(f(x+\tau)-c(x, t)) X_{1}+D X_{1, x x} \\
& X_{2, t}=r(f(x-\tau)-c(x, t)) X_{2}+D X_{2, x x}
\end{aligned}
$$

on a finite interval $-1<x<1$, with $f$ chosen to be the Gaussian $f(x)=\exp \left(-x^{2} / \sigma^{2}\right)$. We impose no flux boundary conditions at $x= \pm 1$. Recall, as previously,

$$
c(x, t)=f(x+\tau) X_{1}+f(x-\tau) X_{2} .
$$

The aim is to illustrate the significance of the length scale $\sqrt{D / r}$ which measures, roughly, the distance that organisms can diffuse before growth effects become important. As $r$ becomes large, this length scale approaches zero and the implicit assumption made in the asymptotic analysis of the dominance of diffusion (see section 2.1.1), becomes invalid.

According to Theorem 2.1 and the results of [14] and consistent with asymptotic predictions, since

$$
\int_{-1}^{1}(f(x+\tau)-f(x-\tau)) d x=0
$$

then both extinction states, $\left(X_{1}=1, X_{2}=0\right)$ and $\left(X_{1}=0, X_{2}=1\right)$, are unstable and there is for each $r>0$ a unique stable coexistence steady state solution to (2.12). We used AUTO [11] to compute the one parameter family (in $r$ ) of these stable 

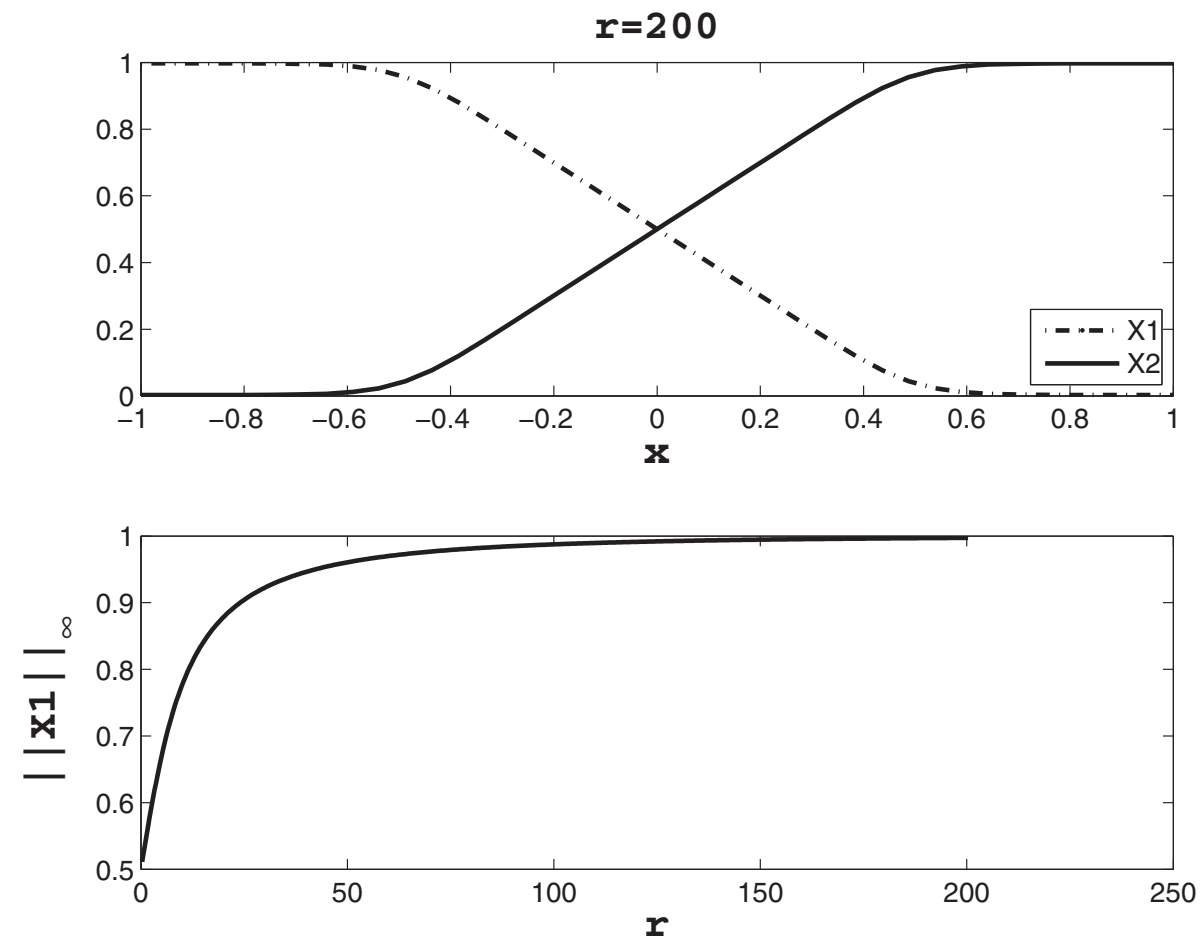

FIG. 2. Stable solution for $r=200$ (top) and steady state solution branch (bottom) for (2.12) with $D=1, \tau=0.6, f(\xi)=\exp \left(-|\xi|^{2} / \sigma^{2}\right)$, and $\sigma^{2}=0.02$.

steady states. In particular, AUTO numerically follows solutions of a finite difference approximation for

$$
\begin{aligned}
& D X_{1, x x}+r\left(f(x+\tau)-f(x+\tau) X_{1}-f(x-\tau) X_{2}\right) X_{1}=0, \\
& D X_{2, x x}+r\left(f(x-\tau)-f(x+\tau) X_{1}-f(x-\tau) X_{2}\right) X_{2}=0 .
\end{aligned}
$$

This also allowed us to compute stability along the branch. Results are shown in Figure 2. Note that at small $r$, both species coexist everywhere in approximately equal concentrations, i.e., $X_{1}$ and $X_{2}$ are close to $1 / 2$ for all $x$, consistent with the asymptotic analysis which predicts that $X_{i}=0.5+\mathrm{O}(r)$. At large $r$, however, the solution approaches the $D=0$ case where, at each location $x$, the more fit species excludes the less fit one, i.e., $X_{1}$ is close to 1 for $x<0$ and $X_{2}$ is close to 1 for $x>0$. This behavior occurs when the distance $\sqrt{D / r}$ becomes small relative to the distance $2 \tau$ between the two species. Note that $\sqrt{D / r}$ would seem to approximate a realized niche width [31] in this model.

This observation suggests that if the two species are far apart (as measured by the ratio of $2 \tau$ and $\sqrt{D / r}$ ), then a third species should be able to coexist between them. To see what happens as this ratio changes we extend to a three species symmetric model,

$$
\begin{aligned}
& X_{1, t}=r(f(x+\tau)-c(x, t)) X_{1}+D X_{1, x x} \\
& X_{2, t}=r(f(x)-c(x, t)) X_{2}+D X_{2, x x} \\
& X_{3, t}=r(f(x-\tau)-c(x, t)) X_{3}+D X_{3, x x}
\end{aligned}
$$

Copyright (C) by SIAM. Unauthorized reproduction of this article is prohibited. 

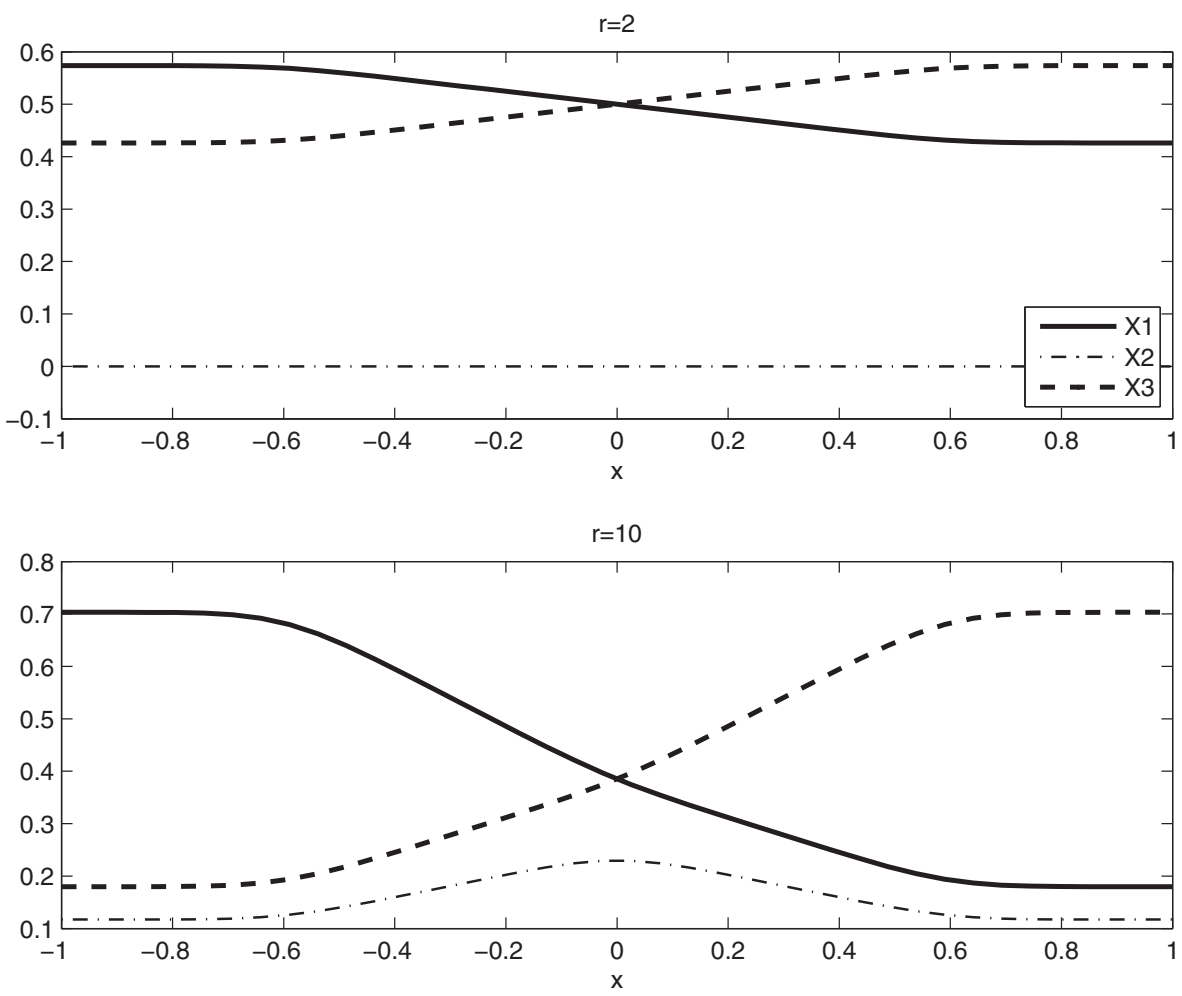

FIG. 3. Stable solutions to (2.14) for $r=2$ and $r=10$. Other parameters are as in Figure 2.

on $-1<x<1$, again with no flux boundary conditions. As before we require that the three species compete for space such that at each point $x$ they satisfy the constraint $X_{1}+X_{2}+X_{3}=1$ with the necessary consequence that

$$
c(x, t)=f(x+\tau) X_{1}+f(x) X_{2}+f(x-\tau) X_{3} .
$$

Results of Theorem 2.1 imply that there are nontrivial steady-state solutions to the system (2.14) for each $r>0$ of the type $X_{i} \equiv 0$ for one $i \in\{1,2,3\}$ at least for some values of $\sqrt{D / r}$, though the stability results are not applicable to the extended three component system. We refer to these states as crowded solutions (since only two of the species are able to coexist, crowding out the third). There can be three such solutions: $\left(X_{1}=0, X_{2} \neq 0, X_{3} \neq 0\right),\left(X_{1} \neq 0, X_{2}=0, X_{3} \neq 0\right)$, and $\left(X_{1} \neq 0, X_{2} \neq 0, X_{3}=0\right)$, though the first and third of these can only exist for sufficiently small $\sqrt{D / r}$; see Theorem 2.1. We have found numerically that the middle stable crowded state is possible. In particular, if $\sqrt{D / r}$ is large enough and hence the realized niche width is large enough, then we can find stable steady state solutions to (2.14) for which the middle species $X_{2} \equiv 0$ while $X_{1}$ and $X_{3}$ are nonzero. That is, due to relatively large diffusivity and no flux boundary conditions, species $X_{1}$ and $X_{3}$ can exclude the intermediate species $X_{2}$. On the other hand, if $\sqrt{D / r}$ is small, then all three species stably coexist. See Figure 3.

We calculated the bifurcation diagram for steady state solutions to system (2.14) numerically using AUTO. AUTO is able to compute the number of unstable eigenvalues for the linearization about our numerical steady state solution to (2.14). This 


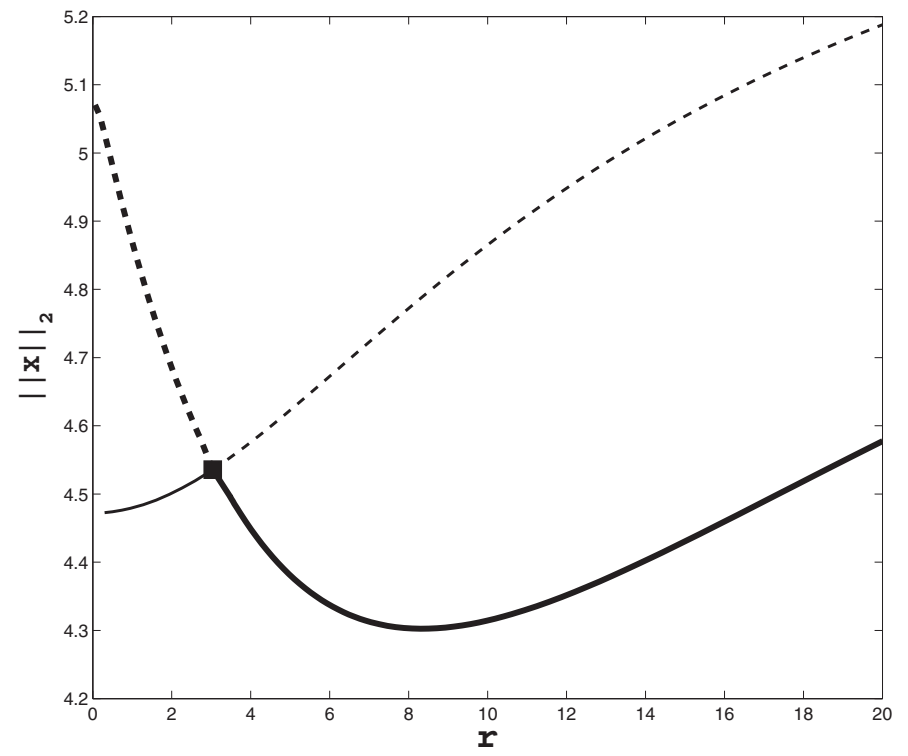

FIG. 4. Bifurcation diagram for steady state solution to (2.14) with $f(\xi)=\exp \left(-|\xi|^{2} /\left(\sigma^{2}\right)\right)$, $\tau=0.6, D=1$, and $\sigma^{2}=0.02$. Vertical axis is the $L_{2}$ norm of the 3-vector of solution components. Horizontal axis measures bifurcation parameter $r$, vertical axis measures solution norm. Solid: stable solutions; dashed: unstable solutions. Thick: two species crowded solutions; thin: three species solutions.

allows us to determine the stability along the solution branches as well as the dimension of the unstable manifold for these steady solution numerical approximations. Partial results are shown in Figure 4. Note again that for small $\sqrt{D / r}$ the crowded solution with middle species $X_{2} \equiv 0$ is stable (see Figure 4, solid black curve), while as $r$ increases, this type of solution loses stability through a transcritical bifurcation at $r \approx 3.03$. Note that at this value of $r$, the ratio $2 \tau / \sqrt{D / r} \cong 2$, that is, the distance between species 1 and species 3 is roughly twice their realized niche widths. For larger values of $r$, when species 1 and species 3 no longer overlap, a stable steady state for which all three species coexist stably emerges (see Figure 4, solid red curve). These results suggest a separation scale on which niche partitioning might occur. In combination with the two species results that indicate that continuum species distribution (i.e., no niche partitioning) can be expected unless the translation invariance of environmental favorability can somehow be broken, we are now prepared to consider the full continuous species model.

We note that the model equations studied in this section are similar to ones that arise in some models of evolution of gene frequencies. Of particular note is the paper [29] which considers a model describing the evolution of gene frequencies in a population subject to migration and selection at a multiallele locus where the selection coefficients are independent of gene frequencies. In the special case where the population density is constant and migration is conservative (does not change density) and isotropic, the equations studied there describing the relative frequencies $X_{i}$ of genotype $A_{i} A_{j}$ at position $x$ at time $t$ are analogous to (2.1) in the case of two alleles and to (2.14) for three alleles. The authors assume genotypic fitnesses that are spatially dependent, corresponding to our functions $f\left(T(x), \tau_{i}\right)$. In much more generality than considered here and for $n$ alleles (see Theorem 1.1 in [29]), they 
prove that $X_{1}(x, t) \rightarrow 1$ uniformly in $x$ if its average fitness (as in (2.4) above but with inequality reversed) exceeds that of all others provided that diffusion $d$ is large relative to scaled fitnesses (a parameter analogous to our $r$ ). In an earlier paper the same authors showed [28, Theorems 1.4 and 1.8] that persistence of all alleles holds and there exists an "internal equilibrium" when diffusion is small relative to $r$ provided that each allele has a selective advantage over all others at some position. Persistence of all alleles means the existence of $\delta>0$ such that $X_{i}(x, t) \geq \delta$ for all $x$ when $t>T$ for some $T$ which may depend on (positive) initial data.

\section{Continuous species distribution.}

3.1. Setup. Consider species volume fraction density $X=X(x, t ; \tau)$ (with units of volume fraction per species), where $-\infty<\tau<\infty$ is the species label, with constraint

$$
\int_{-\infty}^{\infty} X(x, t ; \tau) d \tau=1 \quad \forall(x, t)
$$

(i.e., total population at a given location $x$ is constrained by available space) and $X \geq 0$. Note that $\tau$ is now allowed to vary continuously, so that a continuum of differing species may be present at each location $x$ and time $t$. We suppose that $X$ satisfies

$$
X_{t}=r(f(T(x) ; \tau)-c(x, t)) X+D X_{x x},
$$

where $T, r, f, D$, and $c$ are as described previously. In particular, $r f(T(x) ; \tau) X(x, t ; \tau)$ is the growth rate of species (density) $\tau$ at location $x$ and time $t$ and environmental condition $T(x)$, while $r c(x, t) X(x, t ; \tau)$ is the corresponding loss rate as a consequence of competition for space with other organisms at the same location. The loss $c$ is computed by integrating (3.2) over all species labels $\tau$ to obtain

$$
c(x, t)=\int_{-\infty}^{\infty} f(T(x) ; \tau) X(x, t ; \tau) d \tau
$$

using $\int_{-\infty}^{\infty} X_{t} d \tau=\int_{-\infty}^{\infty} X_{x x} d \tau=0$ as consequences of (3.1). We assume that $f \geq 0$ and

$$
\int_{-\infty}^{\infty} f(T(x) ; \tau) d \tau<\infty
$$

Observe that (3.2) has the appearance of being a nonlocal Fisher (or Fisher-KPP) equation $[4,16,17,18,24]$, though the nonlocality in (3.2) is through the species variable $\tau$ rather than the spatial direction $x$ (the direction in which diffusion is active). Also, note that the nonlocal term is effectively an average of fitness $f$ against kernel $X$ rather than the average of $X$ against some sort of competition kernel as typically used in nonlocal Fisher models. One consequence is that the coefficient of $X$ in (3.2) likely changes sign (as a function of $x$ ) so that traveling waves are generally not expected.

3.2. Continuum species equilibrium. We study the problem first on the infinite interval $x \in(-\infty, \infty)$. However, the same (actually easier) analysis works on the finite interval with Neumann or periodic boundary conditions. Consider the special case that $T=x$ and $f=f(T-\tau)=f(x-\tau)$, where $f$ satisfies

$$
f(x) \geq 0, x \in \mathbb{R}, \text { and } 0<\int_{-\infty}^{\infty} f(s) d s<\infty .
$$


Typically, although not necessarily, $f$ assumes its maximum at zero meaning that $x=$ $\tau$ is the most favorable location for the subpopulation experiencing growth function $f(x-\tau)$.

We can look for an infinite species solution of the form $X=X(T(x)-\tau, t)=$ $X(x-\tau, t)=X(\xi, t)$, where $\xi=x-\tau$. By (3.1), this requires that $X(\xi, t)$ is integrable in its first variable. Note then that $c$ is independent of $x$, with $c$ given by

$$
c(t)=\int_{-\infty}^{\infty} f(x-\tau) X(x-\tau, t) d \tau=\int_{-\infty}^{\infty} f(\xi) X(\xi, t) d \xi,
$$

in order to enforce (3.1), and that $X(\xi, t)$ satisfies

$$
X_{t}=r(f(\xi)-c) X+D X_{\xi \xi} .
$$

An equilibrium solution of (3.5) is a positive integrable solution $X(\xi)$ of

$$
D X_{\xi \xi}+r f(\xi) X=r \lambda_{X} X,
$$

satisfying

$$
\lambda_{X}=\int_{-\infty}^{\infty} f(\xi) X(\xi) d \xi, \quad 1=\int_{-\infty}^{\infty} X(\xi) d \xi .
$$

THEOREM 3.1. Under the condition that $\int\left(1+x^{2}\right) f(x) d x<\infty$, there exists a unique equilibrium solution of (3.6)-(3.7).

This result follows from recasting the problem as a linear eigenvalue problem; see the appendix for details. The solution $X(\xi)=X(x-\tau)$ of (3.6)-(3.7) sets the species distribution at a location $x$. A full continuum of species are able to coexist at each spatial location and tends to zero as $\tau \rightarrow \pm \infty$. This distribution shifts continuously in $\tau$ as $x$ varies. Note again that $\sqrt{D / r}$ sets the natural length scale of (3.5) and hence also the width of the distribution $X$.

3.3. Numerics. We computed solutions to (3.1)-(3.2) (or to (3.6)-(3.7)) on a space-species rectangular domain $x \in[-L L], \tau \in[-L-H L+H]$ with $T(x)=x$ for several choices of environmental response function $f$ and for two different choices of spatial boundary conditions (periodic and no flux, to be discussed in detail below). Spatially periodic boundary conditions were used to approximate infinite spatial domains. Each of our choices for $f$ had an approximate width $w$ in the sense that $f(T ; \tau)$ is small for $|T-\tau|>w$ with $w$ small compared to $L$; in the case of no-flux boundary conditions, the parameter $H$ was chosen to be sufficiently larger than $w$ to ensure that all species that are capable of being competitive within the spatial domain are present. For periodic boundary conditions, $H=0$ was appropriate; see below.

The rectangular domain was discretized sufficiently finely so as to resolve both the response function width $w$ and the length $\sqrt{D / r}$. The resolution of the discretization was tested by grid refinement in a number of cases. Equation (3.2) was integrated using a modified Crank-Nicolson method with growth terms treated explicitly. The loss rate $c(x, t)$ was computed by approximating (3.3) through summing the discretized version of (3.2) over the species label $\tau$. Although this summation leads to a first order approximation of the integral in (3.3), it also satisfies the constraint (3.1), which is anyway the true purpose of the loss term, to round-off error. Initial conditions for all computations shown were chosen to be uniformly constant, i.e., initial species density at a grid location $x_{i}, \tau_{j}$, was set to be $X\left(x_{i}, 0 ; \tau_{j}\right)=C$, where $C=(2(L+H))^{-1}$ to satisfy constraint (3.1). We have also sometimes used random initial conditions to check some results. 

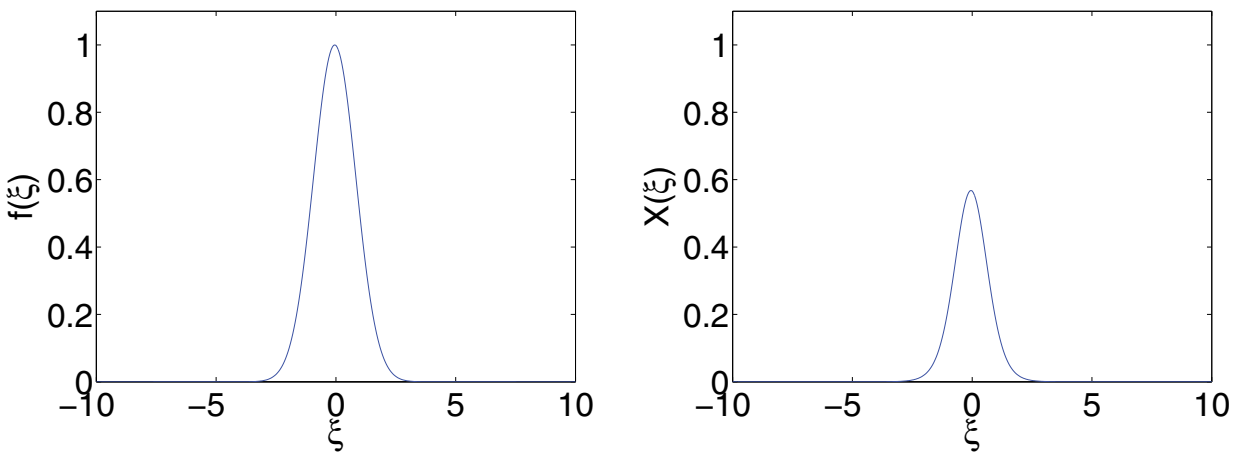

FIG. 5. Left panel: one period of the $2 L$ periodic extension of the temperature response function $f(\xi)=\exp \left(-|\xi|^{2} /(\pi / 2)\right)$. Right panel: one period of the resulting $2 L$ periodic species volume fraction $X(\xi, t)$ at $t=10^{4}$.

3.3.1. Spatially periodic boundaries: Translational equivalence. In order to approximate an infinite spatial domain $x \in(-\infty \infty)$, we impose periodic boundary conditions on the computational domain $x \in(-L L)$ and choose $f(T ; \tau)$ to be $2 L$ periodic in $T(x)$, where $L$ is chosen to be much larger than the width $w$ of $f$ as well as $\sqrt{D / r}$. While the periodic bump structure of the environmental response function is nonrealistic, the bumps are sufficiently far from each other so that in practice they do not feel each others' effects in the computations (except when $T$ is close to $L$ or $-L$ where, as intended, the periodicity "fools" the local population into thinking there is no boundary).

As a representative example, we choose $f=f(\xi)=\exp \left(-|\xi|^{m} / 2 \pi \sigma^{2}\right)$ for $\xi \epsilon$ $\left[\begin{array}{ll}-L & L\end{array}\right]$ with $L=10, \xi=x-\tau$, and $m=2, \sigma=0.5$, see Figure 5, left panel. $2 L$ periodicity defines $f$ outside of the interval $[-L L]$. Other parameter values are $D=0.1, r=1, d x=d \tau=0.05$. Different choices of parameter values (including $m$ smaller or larger than 2) do not appear to result in qualitatively different solutions. With these choices, $X(\xi, t)$ reaches a steady state shown in Figure 5, right panel. This computed $X$ satisfies (3.6)-(3.7) (with central differencing approximation to the second derivative) with residual of $10^{-12}$ or less, pointwise. Contours of the solution $X$ in $x, \tau$ coordinates are shown in Figure 6. Note that the species structure is the same at each $x$ except translated in $\tau$. That is, the continuously varying species solution is apparently stable. The numerical solution is periodic; in the full plane, the contour plot would consist of a tiling using the square shown in Figure 6 with repeating, parallel (with slope 1) clusters of contour lines. In between the clusters, however, $X$ is vanishingly small and there is effectively no diffusive communication between clusters.

In reality, tolerance functions cannot necessarily be expected to have the symmetry of Gaussians. Hence we also tried the function

$$
f(\xi)= \begin{cases}0, & \xi<\xi_{\min } \\ b\left(\xi-\xi_{\min }\right)^{2}\left(1-\exp \left[d\left(\xi-\xi_{\max }\right)\right]\right)^{2}, & \xi_{\min } \leq \xi \leq \xi_{\max } \\ 0, & \xi>\xi_{\max }\end{cases}
$$

(see Figure 7), a form that has been fitted to growth versus temperature data for a large variety of bacteria, e.g., [43]. We chose $d$ so that the tolerance maximum was $3 / 4$ of the way from the minimum tolerable environmental variation $\xi_{\min }$ to the maximum tolerable environmental variation $\xi_{\max }$, in rough agreement with [43]. The parameters 


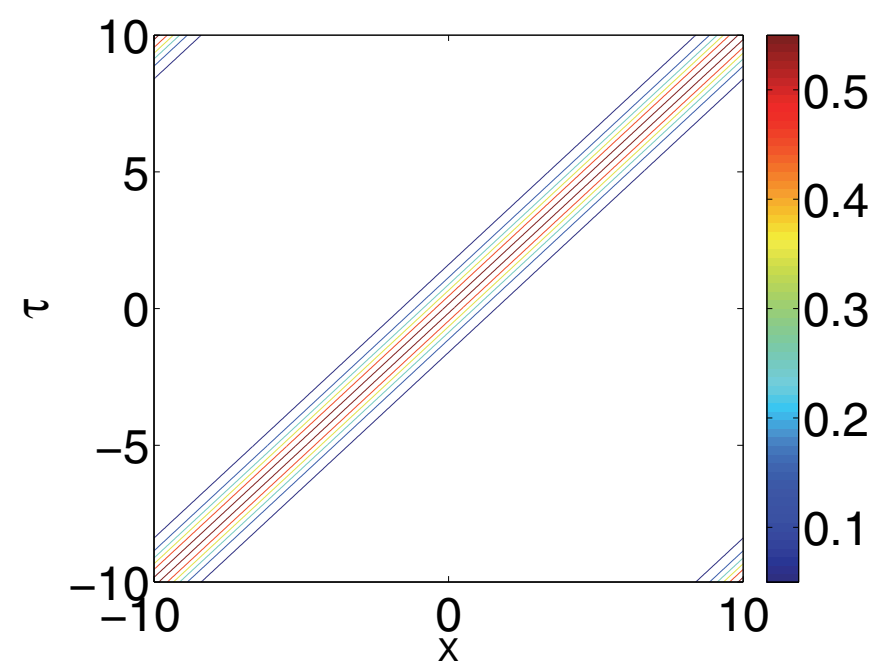

FIG. 6. Contour plot of $X(x, t ; \tau)$ for $t=10^{4} . D=0.1, r=1$.
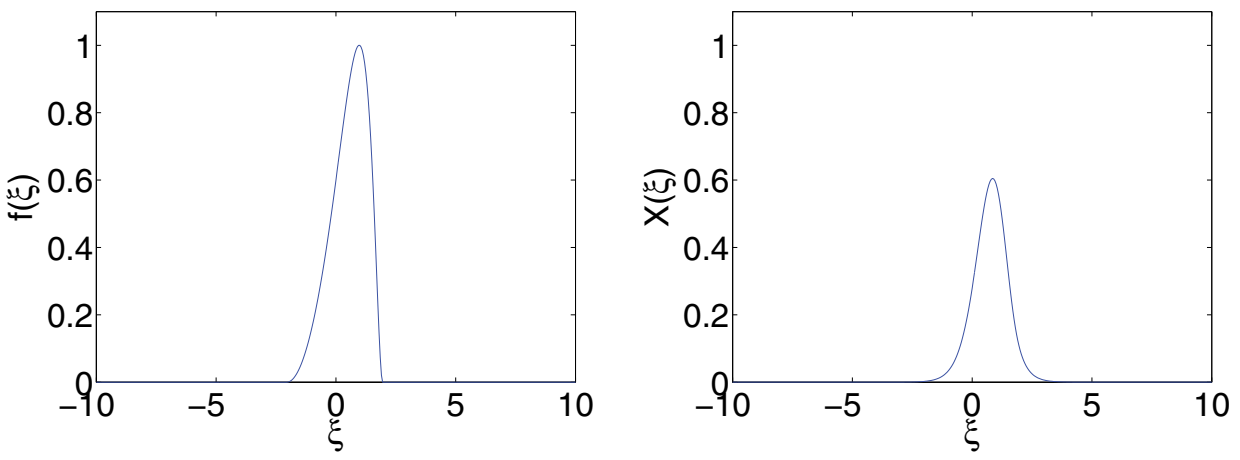

FIG. 7. Left panel: one period of the $2 L$ periodic temperature response function given in (3.8) with $b=(1 / 9)\left(1-e^{-2}\right)^{-2} \approx 0.1486, d=2, \xi_{\min }=-2, \xi_{\max }=2$. Right panel: one period of the resulting $2 L$ periodic species volume fraction $X(\xi, t)$ at $t=10^{4}$.

$b, \xi_{\min }, \xi_{\max }$ were chosen so that the height and width of $f$ would be approximately the same as the Gaussian version; see Figure 5, left panel. Results (contour plot not shown) did not vary qualitatively; compare Figures 5 and 7, right panels.

Altogether, numerics seem to indicate that for translationally equivalent environmental responses, i.e., for $f=f(\xi)$, there is no mechanism for discrete species structure to emerge. That is, the balance of diffusion and environmentally controlled growth with spatial competition does not result, in and of itself, in a mechanism for discrete niche partitioning. This conclusion is supported by the asymptotics of the two species (with species labels $\tau_{1}$ and $\tau_{2}$ ) case, where for any applicable $f=f(\xi)$ and fixed $r$ and in the limit of $\tau_{1}$ approaching $\tau_{2}$, the two species are able to coexist.

3.3.2. Spatially periodic boundaries: Translational modulation. In environmental systems, a translationally equivalent environmental response on an infinite interval does not occur. We thus will remove both of these assumptions starting with translational equivalence $f=f(\xi)$. Translational equivalence implicitly supposes 


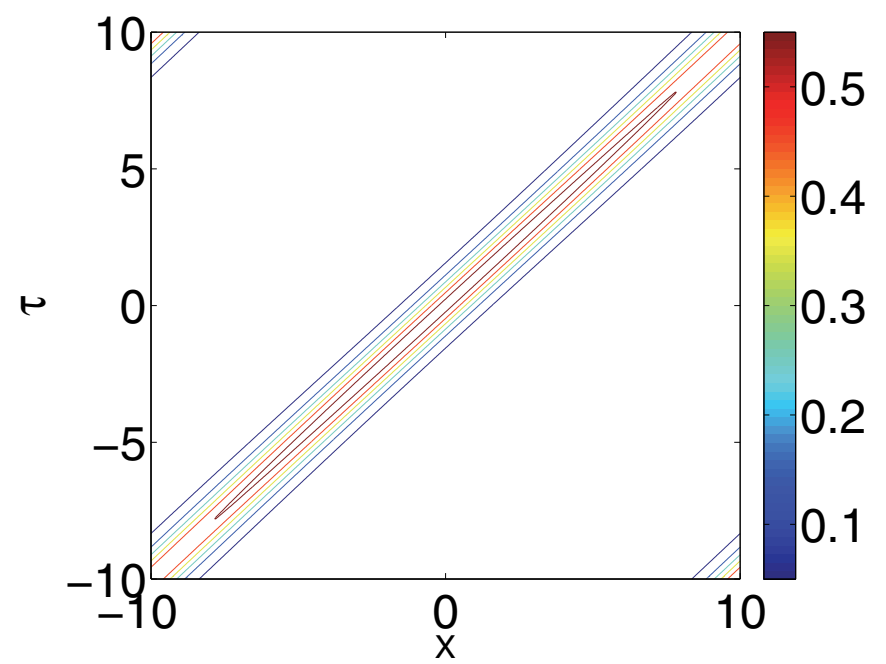

FIG. 8. Contour plot of $X(x, t ; \tau)$ for $t=10^{4}$ with $f(x ; \tau)=(1+0.1 \cos (\pi \tau / 10))$ $\exp \left(-|x-\tau|^{2} /(\pi / 2)\right)$. Parameters are the same as for the computation illustrated in Figure 6.

that all environmental conditions are equally favorable. By relaxing this assumption we can test the robustness of the partitioning model to more realistic environmental influence - perhaps differences, even small ones, in environmental favorability can trigger finite niche partitioning? Note that without translational equivalence, $f=f(T(x) ; \tau)$ need not satisfy $\left\langle f_{\tau}\left(T(x) ; \tau_{0}\right)\right\rangle=0$, even over an infinite interval, so that, in the two species setup anyway, one species may be more likely to outcompete the other asymptotically as their preferred temperatures come together.

As a particular form, we considered a modulated environmental response function of the type $f=\alpha(x) g(x-\tau)$. Recalling the interchangeability of spatial coordinate $x$ and environmental condition $T$, we can think of the amplitude modulator $\alpha$ as, for example, picking out particularly good or bad environmental conditions. For a first example, we add a relatively small amplitude, slow modulation to the temperature response function used in Figure 6 by setting $f(x ; \tau)=(1+0.1 \cos (\pi \tau / 10))$ $\exp \left(-|x-\tau|^{2} /(\pi / 2)\right)$. This response function favors those species with labels $\tau$ such that $\cos (\pi \tau / 10)$ is close to 1 and, conversely, disfavors those species with labels $\tau$ such that $\cos (\pi \tau / 10)$ is close to -1 . Otherwise, parameters are the same as those used in the computation for Figure 6 . Contours of the computed solution for $X(x ; \tau)$ are shown in Figure 8 and, although modulated, are also similar to those in Figure 6. This example together with similar computations suggests that long wavelength perturbations of translational invariance do not result in species clumping.

As a second example, we use a relatively rapid modulation to the temperature response function by setting $f(x ; \tau)=(1+0.1 \cos (10 \pi \tau)) \exp \left(-|x-\tau|^{2} /(\pi / 2)\right)$. Again, other parameters are the same as those used in the computation for Figure 6. Contours of the computed solution for $X(x ; \tau)$ are shown in Figure 9. In this instance, we see the species structure is discretized into ten species per period (one for each crest of the modulation). Figure 10 presents cross sections of $X$ : the left panel shows the species structure at a fixed location and the right panel shows the distribution in $x$ of an individual, surviving species. The difference between the examples of Figures 8 and 9 is quantified by the length scale $\sqrt{D / r}$ which measures, roughly, how 


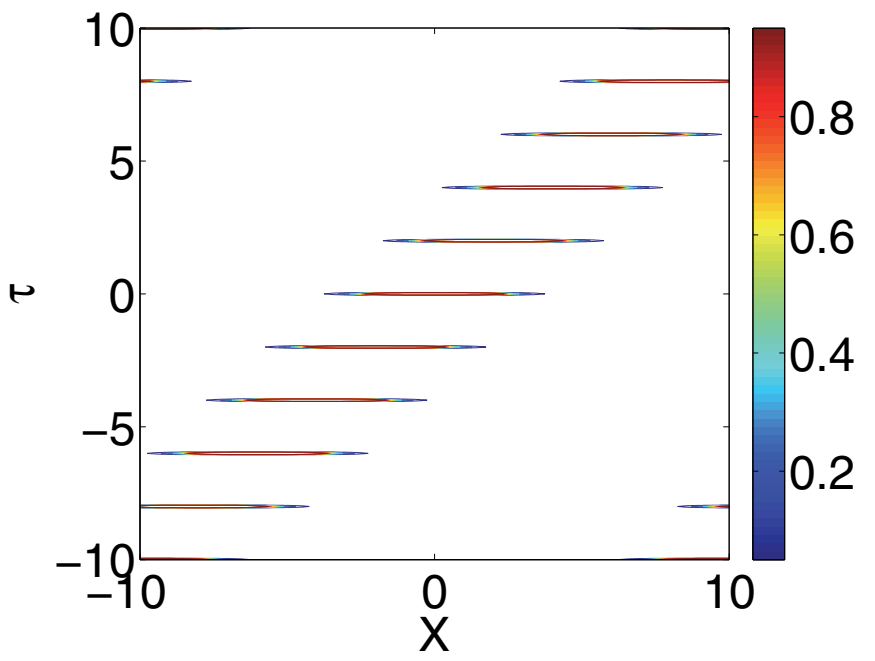

FIG. 9. Contour plot of $X(x, t ; \tau)$ for $t=10^{4}$ with $f(x ; \tau)=(1+0.1 \cos (10 \pi \tau))$ $\exp \left(-|x-\tau|^{2} /(\pi / 2)\right)$. Parameters are the same as for the computation illustrated in Figure 6. Discretization of species structure is indicated-projecting contours onto the $\tau$-axis would show that most species are absent (or nearly so) and the remaining species are separated by gaps (in $\tau$ ). Projecting onto the $X$-axis indicates that, roughly, three or four species predominate at each spatial location.
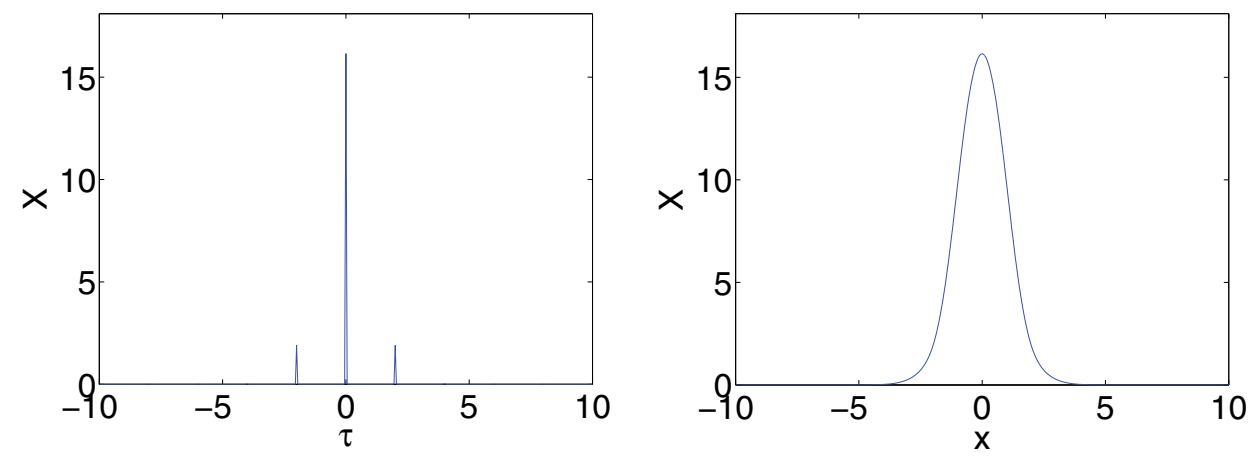

Fig. 10. Left panel: cross section of $X$ (as shown in Figure 9) as a function of $\tau$ at $x=0$. The three spikes mark the three species labels that happen to predominate at location $x=0$. Right panel: cross section of $X$ (as shown in Figure 9) as a function of $x$ at $\tau=0$. This curve shows the niche interval of the species label $\tau=0$.

far organisms can drift diffusively within the time scale of significant growth. For the computation shown in Figure 8, the ratio of perturbation length scale to $\sqrt{D / r}$ is approximately 63 . In the case of Figure 9 this ratio is about 0.63 . Generally, environmental modulations with shorter wavelengths than $\sqrt{D / r}$ can be felt by the organisms resulting in clumping; modulations with longer wavelengths are effectively invisible.

3.3.3. No-flux boundaries. Spatially infinite environments do not exist as a rule. So, while their use has been a convenient way to isolate interactions of growth and mobility from the influence of boundary effects, it is interesting to consider a finite domain $x \in[-L, L]$ with no-flux boundary conditions (microbes cannot escape 


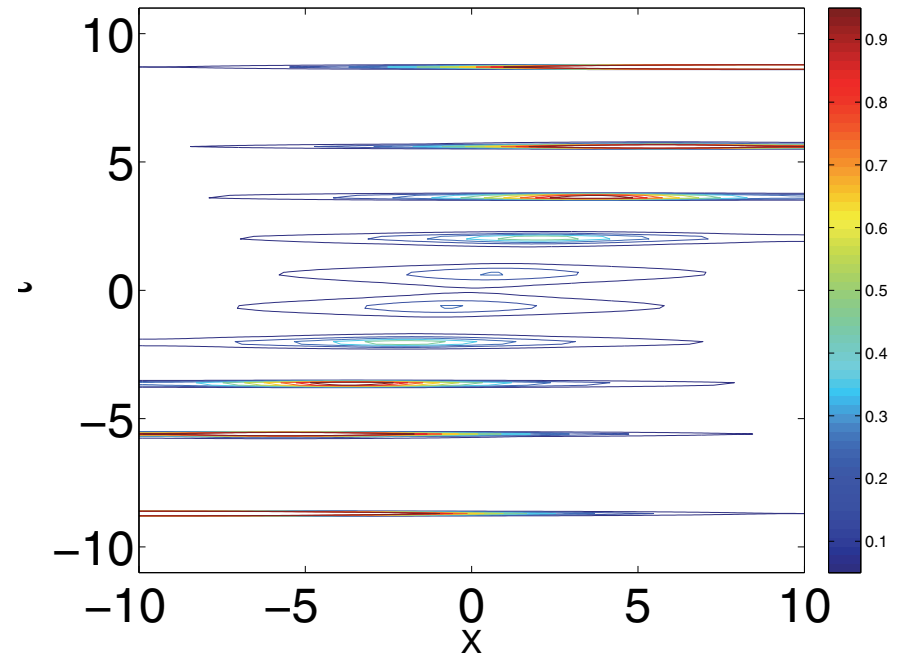

FIG. 11. Contour plot of $X(x, t ; \tau)$ for $t=3.0 \cdot 10^{6}$. As in Figure 9, projecting contours onto the $\tau$-axis indicates that discretization in species space is occurring.
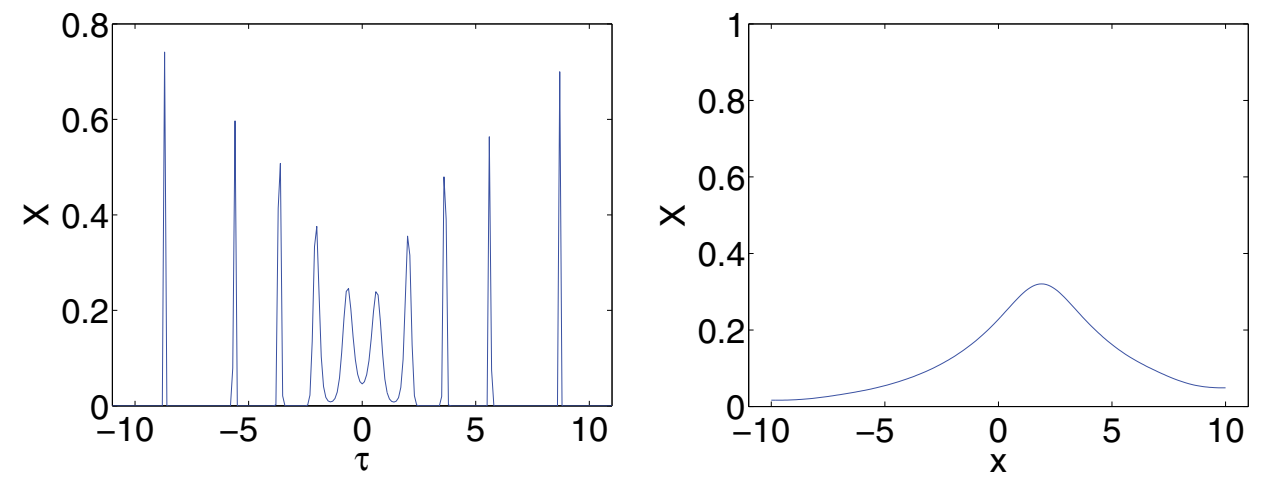

Fig. 12. Left panel: vertical cross section of $X$ (as shown in Figure 11) as a function of $\tau$ at $x=0$. The spikes indicate species labels that happen to predominate at location $x=0$. Right panel: horizontal cross section of $X$ (as shown in Figure 11) as a function of $x$ at $\tau=2$. The curve describes the niche of species label $\tau=2$.

the domain) as in section 2.2. This is a significant perturbation (see Figures 11, 12, and 13): species optimally preferring environmental conditions close to (but inside) those existing at one of the boundary endpoints are subject to less competitive stress than the infinite interval case, because species optimally preferring conditions nearby (but outside) the boundary endpoints are relatively disadvantaged. This special advantage enables them to largely control a territory near the domain boundaries and crowd out nearby species. Towards the edge of this territory in the domain interior, however, these species become less dominant, effectively creating a new, smaller boundary-like region where new dominant species can emerge; see, for example, Figure 13(a)-(b) where the smaller subinterval is, roughly, $[-77]$. Another discretization instability then occurs near the boundaries of this subinterval; see Figure 13(c)-(d). This process iterates until the entire domain is thus carved up; see Figure 13(e)-(h). 
(a) $t=10$

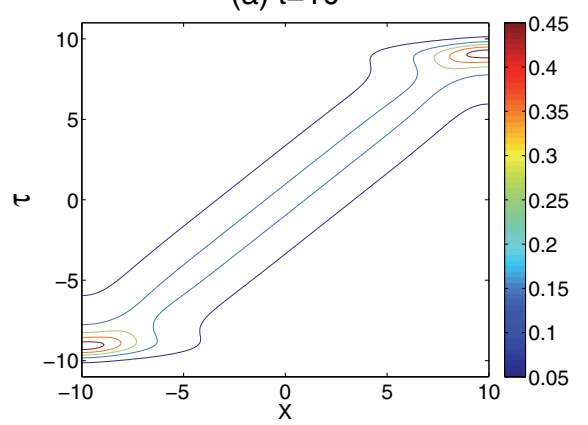

(c) $t=1000$

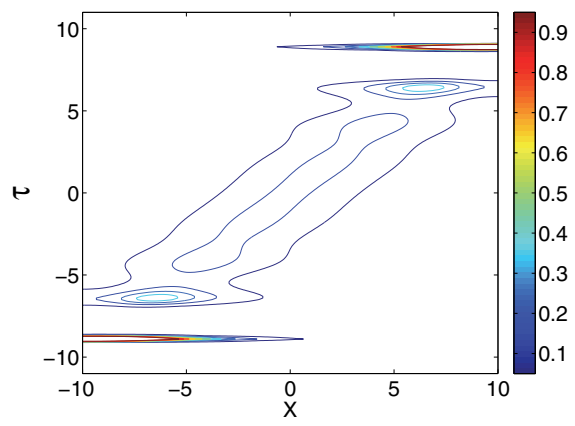

(e) $t=10^{5}$

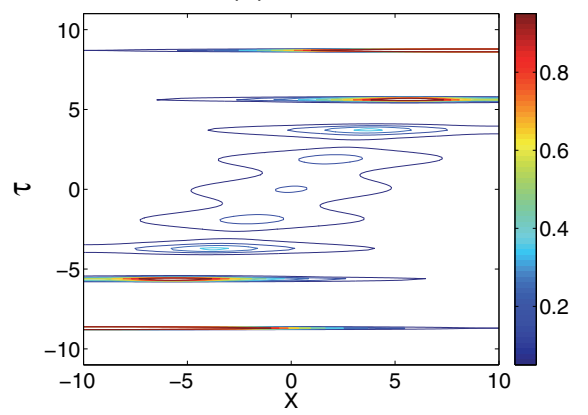

(g) $t=1.5 \times 10^{6}$

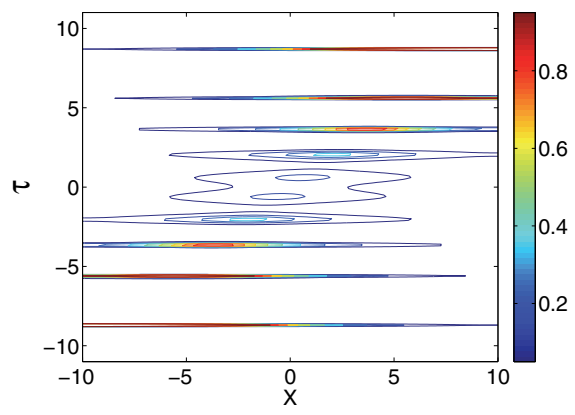

(b) $t=100$

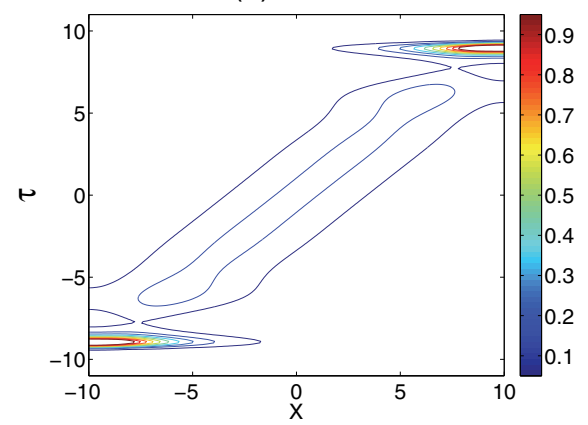

(d) $t=10^{4}$

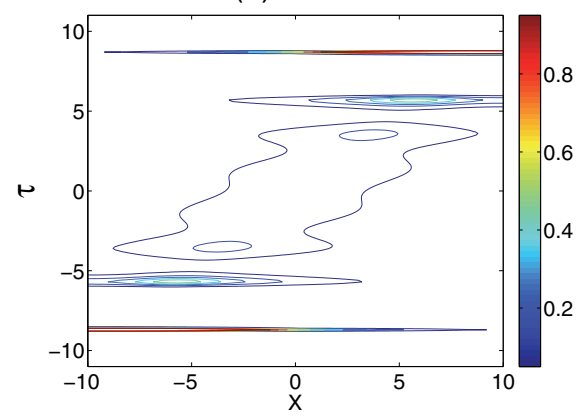

(f) $t=5 \times 10^{5}$

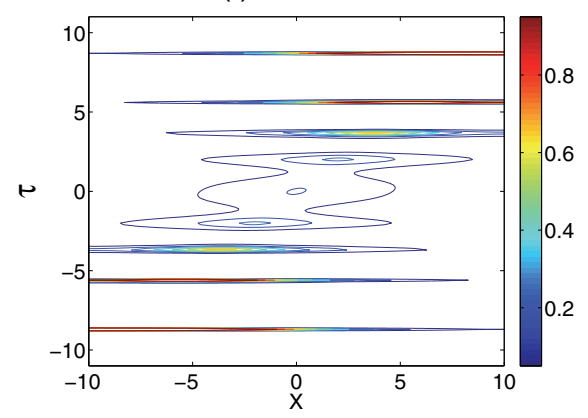

(h) $t=3.0 \times 10^{6}$

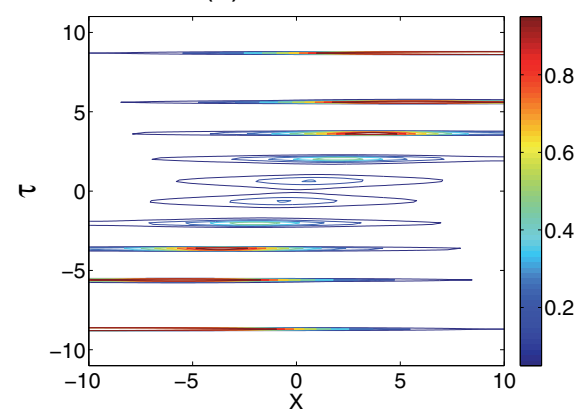

FIG. 13. Time series for the same computation as in Figures 11 and 12. Projecting onto the $\tau$-axes indicates that while all species in the range, roughly, $-10 \leq \tau \leq 10$ are present initially, over time discretization occurs working from the outside species inwards.

Copyright $@$ by SIAM. Unauthorized reproduction of this article is prohibited. 
Parameters for Figures 11-13 are $D=3, r=1, f$ is a Gaussian with width 0.5. It is also worth noting that the latter stages of discretization occur very slowly, so slowly in fact that the time scale might, at some point, overlap with that of evolutionary change [38].

4. Discussion. Through use of highly conserved genetic sequences, e.g., those coding for bacterial 16S rRNA [49], as species classification tools, researchers are currently surveying microbial community structure in many contexts. Recently, careful examination of putative niche partitioning in spatially varying environmental conditions has suggested that, rather than continuous variation, instead finite and discrete (in species space) species structure is observed [7, 22, 33, 37, 42]. Classification based on gene sequence has in at least one instance been supported by matching phenotypic differences with respect to temperature tolerance in a Yellowstone hot spring effluent channel $[3,13]$. In that particular system, other than an inflow boundary, there are no obvious, abrupt coincident environmental changes to explain species demarcations. Further, the channel is sufficiently irregular and complex so that isolation of factors that determine species discretization and niche width is difficult. As such empirical studies become more common for more and more complex systems, it seems likely that simplified, theoretical models of the type presented here may be useful or even essential in suggesting hypothetical mechanisms for determination of community ecological structure at the species level. Generally, one might like to understand the relation between any particular environment and the species structure that is present. This model is meant as a step in that direction.

In fact, the notion of a microbial species in isolation from its environment is not even necessarily a clear one. The concept of species is accepted for organisms that reproduce sexually, largely because any one species is usually reproductively isolated from members of different species; for example, the biological species definition groups together organisms that exchange genetic information among themselves [32, 9]. Such does not apply, however, for asexually reproducing microbial organisms which, on the one hand, reproduce clonally and, on the other, can often exchange genetic material with other, unrelated microorganisms. Some suggest that, as a consequence of such lateral gene transfer and other available genetic manipulation mechanisms, coherent microbial species may not even exist in many instances [12]. One can rather imagine that fluidity of genetic content might result in collections of organisms selected for the local conditions, at least in a stable environment. If environmental conditions vary spatially, then, the result would be something like a continuously varying genome distribution. The aim here is to investigate the stability of such an ecology. Might a continuously varying genome spontaneously break up into a discrete set of clumps which could be viewed as a discrete set of species, that is, is there an inherent tendency for discrete niche partitioning? The presented model suggests that though this may not occur in an ideally varying environment or, at least, no mechanism for discretization has been found under the model assumptions, nevertheless such an instability might well occur under reasonable environmental inhomogeneities - even imposition of boundary conditions can be a trigger, for example. Clumping can be interpreted as development of a discrete species structure. And, while the full development of the instability might be a slow process, the initial breakup can be rapid enough that clumping and thus niche partitioning can be predicted even in time varying environments. That is, the message of the model studied here is that, while there is no apparent inherent mechanism for species discretization, nevertheless natural environmental heterogeneities (like boundaries) can trigger such a discretization. The key 
length scale, at least in the model, is determined by $\sqrt{D / r}$, the length that organisms diffuse before growth effects become important. (In the case of an advective transport mechanism, e.g. [15], this length scale would presumably be determined rather by $v / r$, where $v$ is a typical advective velocity.) As a reminder, we have conflated length and environmental condition, essentially setting them equal in some sense. If we were to separate them again, then the conversion factor $\alpha$ (see section 2) determines the discretization distance in species space as $\alpha \sqrt{D / r}$.

The viewpoint offered here differs a bit from the traditional focus on biological processes as instruments for amplification or suppression of genes and genomes and rather is meant to remind one of the importance of environment in structuring ecologies independent of particular mechanisms. That is, how does the environment itself dictate its ecology? (Such types of questions fit under the rubric of landscape ecology [48].) Ultimately, the goal might be to predict at least some aspects of local ecology based on knowledge of local environment. Though it is likely unrealistic to expect to predict all ecology independent of the details of genetic manipulation processes in all cases, it is still possible that environmental effects are evinced through species structure. More broadly, fundamental questions of microbial community function and efficiency are closely tied to community structure (e.g., clumped versus continuous species distribution) as well as species structure (e.g., realized niche width), and both of these are closely tied to environmental structure. Models of the type proposed here can and are meant to aid in development of the basic intuition and theory needed to formulate hypotheses just as they have been able to do so for plant and animal communities. In light of rapid advances in observational microbial ecology, such theory is timely.

Appendix: Proof of Theorem 3.1. Let $u$ be a nontrivial positive solution of the linear eigenvalue problem

$$
\mu u^{\prime \prime}+f(s) u=\lambda u,
$$

where $\mu=D / r, f$ satisfies (3.4), eigenvalue $\lambda \in \mathbb{R}$, and

$$
\int_{-\infty}^{\infty} u(s) d s<\infty
$$

We then say that $u$ is a principal eigenfunction corresponding to the principal eigenvalue $\lambda$. Note that a nontrivial positive solution of (4.1) satisfies $u(s)>0, s \in \mathbb{R}$.

LEMma 4.1. There is at most one principal eigenvalue $\lambda$ and the corresponding principal eigenfunction $u$ is unique up to positive multiple. Moreover, $0<\lambda<\|f\|_{\infty}$. $u$ satisfies $u(s) \sim e^{-|s| \sqrt{\lambda / \mu}}$ as $s \rightarrow \pm \infty$.

Proof. The positivity and integrability of $u$ are easily seen to imply that $0<\lambda<$ $\max f$. Otherwise, the second derivative of $u$ has a fixed sign which is incompatible with positivity and integrability.

As $\lambda>0$ and $f$ is integrable, the differential equation (4.1) has a fundamental set of solutions $u_{1}, u_{2}$ satisfying $u_{1}(s) \sim e^{-s \sqrt{\lambda / \mu}}$ as $s \rightarrow \infty$ and $u_{2}(s) \sim e^{s \sqrt{\lambda / \mu}}$ as $s \rightarrow \infty$ by Theorem 2 of Chapter IV of [8]. Integrability of a principal eigenfunction $u$ implies that $u$ is a multiple of $u_{1}$. A similar argument applies as $s \rightarrow-\infty$, so $u(s) \sim e^{-|s| \sqrt{\lambda / \mu}}$ as $s \rightarrow \pm \infty$.

If both $\lambda$ and $u$ and $\tilde{\lambda}$ and $v$ are principal eigenvalue and principal eigenfunctions, respectively, then multiplying (4.1) by $v$ and integrating yields 


$$
\begin{aligned}
\lambda \int_{-\infty}^{\infty} u(s) v(s) d s & =\int_{-\infty}^{\infty} v(s)\left(\mu u^{\prime \prime}(s)+f(s) u\right) d s \\
& =\int_{-\infty}^{\infty} u(s)\left(\mu v^{\prime \prime}(s)+f(s) v\right) d s \\
& =\tilde{\lambda} \int_{-\infty}^{\infty} u(s) v(s) d s
\end{aligned}
$$

implying that $\lambda=\tilde{\lambda}$. As both $u$ and $v$ must be multiples of $u_{1}$, it follows that they are positive multiples of each other.

Proposition 4.2. Let $f$ satisfy (3.4). Then there exists a positive solution of (3.6) and (3.7) if and only if (4.1) has a principal eigenfunction. There exists at most one positive solution of (3.6) and (3.7).

Proof. If (3.6) has a positive solution $X(\xi)$ satisfying (3.7), then $u=X$ is a principal eigenfunction of (4.1).

Conversely, if $u$ is a principal eigenfunction of (4.1), then integrating the equation and applying the normalization $\int_{-\infty}^{\infty} u(s) d s=1$ yields that

$$
\int_{-\infty}^{\infty} f(s) u(s) d s=\lambda
$$

Therefore, we see that a principal eigenfunction $u$ yields an equilibrium solution $X(\xi)=u(\xi)$ of $(3.6)$.

The uniqueness assertion follows from the uniqueness of the normalized principal eigenfunction of (4.1).

The existence of a principal eigenfunction and principal eigenvalue of (4.1) is a classical problem for the one dimensional Schrödinger equation, where, traditionally, (4.1) is multiplied by -1 and where $-f(s)$ is regarded as the potential function. See Theorem 2.5 of [45].

THEOREM 4.3. Let $f$ satisfy the hypotheses of Proposition 4.2 as well as $\int\left(1+x^{2}\right) f(x) d x<\infty$. Then there exists a principal eigenvalue and principal eigenfunction of (4.1).

A useful result from Simon's paper [45, Theorem 2.5], is that if $\int_{\mathbb{R}}\left(1+x^{2}\right) \beta(x) d x<$ $\infty$, then $\frac{d^{2}}{d x^{2}}+\beta(x)$ has a positive eigenvalue if and only if $\int_{\mathbb{R}} \beta(x) d x \geq 0$. See also Theorem XIII.110 in [44]. The restriction $\int\left(1+x^{2}\right) f(x) d x<\infty$ is unnecessary according to a personal communication from Y. Pinchover. Ideas from [40, 41] and from "criticality theory" can be used to remove the condition.

Acknowledgments. The authors would like to thank Dave Ward for helpful conversations, Yehuda Pinchover and Friedrich Geztesy for useful help, and also to acknowledge support from NSF grants DMS 1022836, DMS 0918440, and MCB 1158553.

\section{REFERENCES}

[1] M. Ballyk And H. Smith, A model of microbial growth in a plug flow reactor with wall attachment, Math. Biosci., 158 (1999), pp. 95-126.

[2] E. W. BEAlS, Vegetational change along altitudinal gradients, Science, 165 (1969), pp. 981-985.

[3] E. D. Becraft, F. M. Cohan, M. Kühl, S. I. Jensen, and D. M. Ward, Fine-scale distribution patterns of Synechococcus ecological diversity in the microbial mat of Mushroom Spring, Yellowstone National Park, Appl. Environ. Microbiol., to appear.

[4] H. Berestycki, G. Nadin, B. Perthame, and L. Ryzhik, The non-local Fisher-KPP equation: Travelling waves and steady states, Nonlinearity, 22 (2009), pp. 2813-2844. 
[5] R. S. Cantrell, C. Cosner, and Y. Lou, Evolution of dispersal in heterogeneous landscape, in Spatial Ecology, Math. Comput. Biol. Ser., eds. R. S. Cantrell, C. Cosner, S. Ruan, Chapman \& Hall/CRC, London, 2009, pp. 213-229.

[6] W. G. Characklis and K. C. Marshall, eds., Biofilms. John Wiley \& Sons, New York, 1990.

[7] N. Connor, J. Sikorski, A. P. Rooney, S. Kopac, A. F. Koeppel, A. Burger, S. G. Cole, E. B. Perry, D. Krizanc, N. C. Field, M. Slaton, and F. M. Cohan, Ecology of speciation in the genus Bacillus, Appl. Environ. Microbiol., 76 (2010), pp. 1349-1358.

[8] W. Coppel, Stability and Asymptotic Behavior of Differential Equations, D.C. Heath, Boston (1965).

[9] K. DE QueIroz, Species concepts and species delimitation, Systems Biol., 56 (2007), pp. 879-886.

[10] J. Dockery, V. Hutson, K. Mischaikow, and M. Pernarowski, The evolution of slow dispersal rates: A reaction diffusion model, J. Math. Biol., 37 (1998), pp. 61-83.

[11] E. J. Doedel And B. E. Oldeman, AUTO-07P: Continuation and Bifurcation Software for Ordinary Differential Equations, http://www.dam.brown.edu/people/sandsted/ auto/auto07p.pdf (2012).

[12] W. F. Doolittle and R. T. Papke, Genomics and the bacterial speciation problem, Genome Biol., 7 (2006), pp. 116.

[13] M. J. Ferris, M. KÜHL, A. Wieland, And D. M. WARd, Cyanobacterial ecotypes in different optical microenvironments of a $68^{\circ} \mathrm{C}$ hot spring mat community revealed by $16 S-23 S$ rRNA internal transcribed spacer region variation, Appl. Environ. Microbiol., 69 (2003), pp. 28932898.

[14] W. H. Fleming, A selection-migration model in population genetics, J. Math. Biol., 2 (1975), pp. 219-233.

[15] R. Freter, Mechanisms that control the microflora in the large intestine, in, Human Intestinal Microflora in Health and Disease, D. Hentges, ed., Academic Press, New York (1983).

[16] J. Furter And M. Grinfeld, Local vs. non-local interactions in population dynamics, J. Math. Biol., 27 (1989), pp. 65-80.

[17] S. Genieys, V. Volpert, And P. Auger, Pattern and waves for a model in population dynamics with nonlocal consumption of resources, Math. Model. Natur. Phenom., 1 (2006), pp. $65-82$.

[18] S. A. Gourley, Travelling front solutions of a nonlocal Fisher equation, J. Math. Biol., 41 (2000), pp. 272-284.

[19] M. Gyllenberg and G. Meszéna, On the impossibility of coexistence of infinitely many strategies, J. Math. Biol., 50 (2005), pp. 133-160.

[20] A. Hastings, Can spatial variation alone lead to selection for dispersal, Theor. Popul. Biol., 33 (1983), pp. 311-314.

[21] F. C. HoppensteAdT, Analysis of a stable polymorphism arising in a selection-migration model in populations genetics, J. Math. Biol., 2 (1975), pp. 235-240.

[22] D. E. Hunt, L. A. David, D. Gevers, S. P. Preheim, E. J. Alm, and M. F. Polz, Resource partitioning and sympatric differentiation among closely related bacterioplankton, Science, 320 (2008), pp. 1081-1085.

[23] G. E. Hutchinson, The paradox of the plankton, Amer. Natur., 95 (1961), pp. 137-145.

[24] P.-E. Jabin And G. RaOul, On selection dynamics for competitive interactions, J. Math. Biol., 63 (2011), pp. 493-517.

[25] I. Klapper and J. Dockery, Mathematical description of microbial biofilms, SIAM Rev., 52 (2010), pp. 221-265.

[26] O. Leimar, M. Doebeli, and U. Dieckmann, Evolution of phenotypic clusters through competition and local adaptation along an environmental gradient, Evolution, 62 (2008), pp. 807-822.

[27] S. A. Levin AND L. A. Segel, Hypothesis for origin of planktonic patchiness, Nature, 259 (1976), p. 659.

[28] Y. LOU AND T. NAGYlaki, Evolution of a semilinear parabolic system for migration and selection in population genetics, J. Differential Equations, 204 (2004), pp. 292-322.

[29] Y. LOU AND T. NAGYlaki, Evolution of a semilinear parabolic system for migration and selection without dominance, J. Differential Equations, 225 (2006), pp. 624-665.

[30] Y. Lou, T. Nagylaki, and W.-M. Ni, An introduction to migration-selection PDE models, Discrete Contin. Dynam. Systems, 33 (2013), pp. 4349-4373.

[31] R. M. MAY AND R. MACARTHUR, Niche overlap as a function of environmental variability, Proc. Natl. Acad. Sci., USA 69 (1972), pp. 1109-1113.

[32] E. MAYR, Systematics and the Origin of Species, Columbia University Press, New York (1942).

Copyright $@$ by SIAM. Unauthorized reproduction of this article is prohibited. 
[33] A. C. Martiny, A. P. K. Tai, D. Veneziano, F. Primeau, and S. W. Chisholm, Taxonomic resolution, ecotypes and the biogeography of Prochlorococcus, Environ. Microbiol., 11 (2009), pp. 823-832.

[34] G. Meszéna, M. Gyllenberg, L. Pásztor, and J. A. J. Metz, Competitive exclusion and limiting similarity: A unified theory, Theor. Pop. Biol., 69 (2006), pp. 68-87.

[35] P. J. Morin, Community Ecology, 2nd ed., Wiley-Blackwell, Hoboken, NJ, 2011.

[36] F. Mizera and G. Meszéna, Spatial niche packing, character displacement and adaptive speciation along an environmental gradient, Evol. Ecol. Res., 5 (2003), pp. 363-382.

[37] R. S. Mueller, V. J. Denef, L. H. Kalnejais, K. B. Suttle, B. C. Thomas, P. Wilmes, R. L. Smith, D. K. Nordstrom, R. B. McCleskey, M. B. Shah, N. C. VerBerkmoes, R. L. Hettich, AND J. F. BANField, Ecological distribution and population physiology defined by proteomics in a natural microbial community, Mol. Systems Biol., 6 (2010), 374.

[38] S. Nee and N. Colegrave, Paradox of the clumps, Nature, 441 (2006), pp. 417-418.

[39] S. Pigolotti, C. López, E. Hernández, and K. H. Andersen, How Gaussian competition leads to lumpy or uniform species distributions, Theor. Ecol., 3 (2010), pp. 89-96.

[40] Y. Pinchover, Maximum and anti-maximum principles and eigenfunctions estimates via perturbation theory of positive solutions of elliptic equations, Math. Ann., 314 (1999), pp. 555-590.

[41] Y. PINChOVER, Topics in the theory of positive solutions of second-order elliptic and parabolic partial differential equations, in Spectral Theory and Mathematical Physics: A Festschrift in Honor of Barry Simons 60th Birthday, B. Simon, F. Gesztesy, eds., Proc. Sympos. Pure Math. 76, Providence, RI, 2007, pp. 329-356.

[42] N. B. Ramsing, M. J. Ferris, And D. M. WARD, Highly ordered vertical structure of Synechococcus populations within the one-millimeter-thick photic zone of a hot spring cyanobacterial mat, Appl. Environ. Microbiol., 66 (2000), pp. 1038-1049.

[43] D. A. Ratkowsky, R. K. Lowry, T. A. McMeekin, A. N. Stokes, and R. E. Chandler, Model for bacterial culture growth rate throughout the entire biokinetic temperature range, J. Bacteriol., 154 (1983), pp. 1222-1226.

[44] M. Reed And B. Simon, Methods of Modern Mathematical Physics IV: Analysis of Operators, Academic Press, New York (1978).

[45] B. Simon, The bound state of weakly coupled Schrödinger operators in one and two dimensions, Ann. Physics, 97 (1976), pp. 279-287.

[46] H. L. Smith and P. Waltman, The Theory of the Chemostat, Cambridge University Press, Cambridge, 1994.

[47] P. S. Stewart and M. J. Franklin, Physiological heterogeneity in biofilms, Nat. Rev. Microbiol., 6 (2008), pp. 199-210.

[48] M. G. Turner, Landscape ecology: the effect of pattern on process, Annu. Rev. Ecol. Systems, 20 (1989), pp. 171-197.

[49] C. R. Woese AND G. E. Fox, Phylogenetic structure of the prokaryotic domain: the primary kingdoms, Proc. Natl. Acad. Sci. USA, 74 (1977), pp. 5088-5090.

Copyright (C) by SIAM. Unauthorized reproduction of this article is prohibited. 